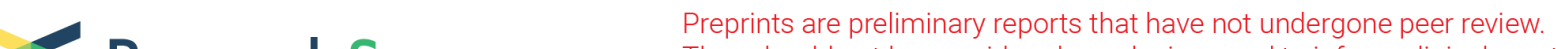

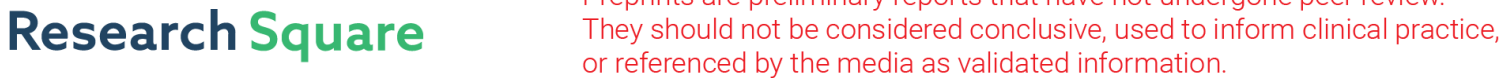

\section{Personalized repetitive transcranial magnetic stimulation for primary progressive aphasia}

\section{Vanesa Pytel}

Hospital Clinico Universitario San Carlos

\section{María Nieves Cabrera-Martín}

Hospital Clinico Universitario San Carlos

Alfonso Delgado-Álvarez

Hospital Clinico Universitario San Carlos

José Luis Ayala

Complutense University of Madrid: Universidad Complutense de Madrid

\section{Paloma Balugo}

Hospital Clinico Universitario San Carlos

\section{Cristina Delgado-Alonso}

Hospital Clinico Universitario San Carlos

\section{Miguel Yus}

Hospital Clinico Universitario San Carlos

\section{María Teresa Carreras}

Hospital Universitario de la Princesa

José Luis Carreras

Hospital Clinico Universitario San Carlos

\section{Jorge Matías-Guiu}

Hospital Clinico Universitario San Carlos

JA Matias-Guiu ( $\nabla$ jordimatiasguiu@hotmail.com )

Hospital Clinico Universitario San Carlos https://orcid.org/0000-0001-5520-2708

\section{Research}

Keywords: primary progressive aphasia, transcranial magnetic stimulation, apraxia of speech, neuromodulation, brain stimulation, frontotemporal dementia

Posted Date: May 6th, 2021

DOI: https://doi.org/10.21203/rs.3.rs-489757/v1

License: (9) (1) This work is licensed under a Creative Commons Attribution 4.0 International License. Read Full License 
Page $2 / 28$ 


\section{Abstract}

\section{Background}

Primary progressive aphasia (PPA) is a neurodegenerative syndrome for which no effective treatment is available. We aimed to assess the effect of repetitive transcranial magnetic stimulation (rTMS), using personalized targeting.

\section{Methods}

We conducted a randomized, double-blind, pilot study of patients with PPA receiving rTMS, with a subgroup of patients receiving active- versus control-site rTMS in a cross-over design. The primary outcome was changes in spontaneous speech (word count). Secondary outcomes included changes in other language tasks, global cognition, global impression of change, neuropsychiatric symptoms, and brain metabolism using FDG-PET.

\section{Results}

Twenty patients with PPA were enrolled (14 with nonfluent and 6 with semantic variant PPA). Compared to the control group, the group receiving active-site rTMS showed improvements in spontaneous speech, other language tasks, patient and caregiver global impression of change, apathy, and depression. This group also showed improvement or stabilization of results obtained in the baseline examination. Increased metabolism was observed in several brain regions after the therapy, particularly in the left frontal and parieto-temporal lobes and in the precuneus and posterior cingulate bilaterally.

\section{Conclusions}

We found an improvement in language, patient and caregiver perception of change, apathy, and depression using high frequency rTMS. The increase of regional brain metabolism suggests enhancement of synaptic activity with the treatment.

\section{Trial registration}

NCT03580954 (clinicaltrials.gov). Registered 10 July 2018 (retrospectively registered).

\section{URL of the trial registry record}

https://clinicaltrials.gov/ct2/show/NCT03580954

\section{Background}

Primary progressive aphasia (PPA) is a clinical syndrome characterized by the neurodegeneration of brain regions and networks involved in language $(1,2)$. As in many other neurodegenerative disorders, the 
available treatments have limited efficacy. Three main variants are currently recognized (the nonfluent, semantic, and logopenic variants), although some additional subtypes have been suggested (3-5).

Transcranial magnetic stimulation (TMS) is a non-invasive brain stimulation (NIBS) technique based on electromagnetic induction (6). Although the precise mechanisms of its effect are largely unknown, TMS induces changes in brain electrical activity and may modulate neuroplasticity and synaptic activity. In post-stroke aphasia, TMS has been shown to improve functional outcomes (7-8). In the context of cognitive neurodegenerative disorders, TMS has shown promising preliminary results in patients with Alzheimer's disease, although large, well-designed clinical trials are still needed (9). In frontotemporal dementia syndromes, TMS has shown positive results for diagnosis (10), but experience with its therapeutic use is very limited (11-12). Most studies of neuromodulation in PPA have used transcranial direct current stimulation (tDCS), an alternative form of NIBS. Using tDCS, several studies have found a certain degree of improvement in specific language tasks (e.g. spelling, naming) by applying stimulation to various targets in the left hemisphere. These targets include the inferior frontal gyrus, dorsolateral prefrontal cortex, and the fronto-temporal and temporo-parietal regions. These studies have generally used a combined approach of tDCS and language therapy, showing a greater improvement with tDCS than with language therapy alone (13-17).

One of the main challenges in NIBS is selecting targets for brain stimulation $(14,18)$. Given the clinical and topographical heterogeneity of neurodegenerative disorders, effective NIBS therapies should probably be personalized to achieve an optimal response. Because of the progressive damage of the language network in PPA, the optimal target should probably be different according to each variant or patient, and the disease stage. Non-fluent PPA is characterized by atrophy and hypometabolism of the left frontal lobe, including the left superior, middle, and inferior frontal, and supplementary motor area. White matter damage involves the left superior longitudinal fasciculus, and also the left inferior frontaloccipital fasciculus, the frontal aslant tract, or left uncinate fasciculus (19). Conversely, in semantic PPA a left predominant anterior temporal atrophy associated with damage of the uncinate fasciculus and the inferior longitudinal fasciculus bilaterally is observed. This impairment reflects the dissociation between the dorsal vs. ventral pathways dysfunction in non-fluent and semantic variants, respectively (20). Furthermore, longitudinal neuroimaging studies have shown heterogeneous regional patterns of progression, especially in the non-fluent variant (21). These patterns of brain damage support the need for searching for different targets for brain stimulation. For instance, in the early stages of PPA a patient could respond to stimulation of one of the hallmark regions at onset (i.e. left anterior temporal lobe in semantic PPA), but in later stages with more severe damage in those regions, alternative targets (prefrontal cortex, or right anterior temporal lobe) could induce greater effects. Thus, we hypothesized that selecting personalized targets for repetitive TMS (rTMS) may induce positive effects in patients with the nonfluent and semantic variants of PPA. Because PPA presents focal onset, with different variants, and cognitive dysfunction is restricted to language impairment, we believe that it may be a good model for evaluating the effect of personalized brain stimulation in neurodegenerative disorders. In this study, we aimed to assess the effect of rTMS with personalized targeting on language, global cognition, brain metabolism, and neuropsychiatric symptoms in patients with nonfluent and semantic variant PPA. 


\section{Methods}

\subsection{Participants}

Twenty patients with PPA were enrolled in this study. All patients met clinical and imaging-supported diagnostic criteria for PPA (1). Inclusion criteria were: i) diagnosis of PPA confirmed with fluorodeoxyglucose positron emission tomography (FDG-PET) imaging; ii) Clinical Dementia Rating scale score of 0-1 (global score); iii) being a native Spanish speaker. The exclusion criteria were as follows: i) any contraindication for TMS or magnetic resonance imaging (MRI); ii) history of epilepsy; iii) other language disorders prior to the diagnosis of PPA; iv) neuroimaging findings not suggestive of PPA. The main clinical and demographic characteristics are summarized in Table 1 and Supplementary Material Table S1. The study was conducted at the Department of Neurology of Hospital Clínico San Carlos, Madrid between February 2018 and February 2020.

\subsection{Study design}

This is a double-blind (participant, outcomes assessors), single-center pilot study of patients with PPA who were treated with rTMS. Simple randomization was used to separate patients into 2 groups with a 3:2 ratio: i) active-site rTMS therapy only $(n=12)$; ii) a cross-over group $(n=8)$. In the latter group, subjects were randomly allocated 1:1 to receive therapeutic rTMS followed by control-site rTMS, or vice versa. Figure 1 summarizes the study design. One patient with semantic variant PPA in the cross-over group dropped out of the study due to a family issue after the active phase was completed; therefore, the final sample consisted of 13 patients in active therapy only, and 7 in the cross-over group. Patients were classified into nonfluent variant PPA ( $n=14,6$ of them in the cross-over group) and semantic variant PPA ( $n=6$, one in the cross-over group) (Figure 2). At the time of study inclusion, patients were evaluated with the Addenbrooke's Cognitive Examination III (22), Progressive Aphasia Severity Scale (23), Functional Activities Questionnaire, and Frontotemporal Lobar Degeneration-modified Clinical Dementia Rating scale (24). All participants were also assessed using a comprehensive battery of speech and language tests, including phonological tasks (initial phoneme deletion, word spelling, non-word repetition), semantic tasks (semantic-association task, word-picture matching, verb-action matching, synonyms judgment), picture object naming, action naming, semantic fluency, letter fluency, action fluency, verbal repetition, and reading of different types of words. Apraxia of speech was evaluated using sequential and alternating dysdiadochokinesia, repetition of multisyllabic words, reading, and spontaneous speech. Other cognitive domains were evaluated using the following tests: Corsi-block tapping, digit span, Trail Making Test, Symbol Digit Modalities Test, Rey-Osterrieth Complex Figure (copy and memory at 3 and 30 minutes), Stroop Color-Word Interference test, Tower of London Drexel version, Visual Object and Space Perception Battery (subtests object decision, progressive silhouettes, position discrimination, and number location), and Judgment Line Orientation. This protocol is described in detail elsewhere (25).

MRI (1.5 T, SIGNA HDxt, GE Healthcare) was performed less than 3 months before the start of the first rTMS session and included the following sequences: T1-weighted 3D fast spoiled gradient-echo, 3D Cube T2-weighted fluid-attenuated inversion recovery, and diffusion-tensor imaging. FDG-PET images (Siemens 
Biograph TruePoint PET/CT scanner) were acquired at rest, according to the European guidelines for brain PET imaging (26). PET images were acquired before the onset and within the first week after the end of each intervention (active-site rTMS and control site rTMS). Language training and medications were not modified during the study. Eight patients (40\%) were receiving active language therapy during the study, with a mean duration of $1.68 \pm 0.59$ hours per week in this group.

\subsection{Target personalization.}

The target for active-site rTMS was selected after a first phase of the study, lasting approximately 10 weeks, in which patients received a single session of rTMS per week using different targets and protocols. Patients received excitatory and/or inhibitory protocols in 6 to 10 different brain targets according to clinical variant and neuroimaging findings. The excitatory protocol consisted of a session of 1500 pulses with $20-\mathrm{Hz}$ rTMS trains with an interval of 20 seconds at $100 \%$ of the resting motor threshold. The inhibitory protocol consisted of a session of 600 pulses with 1-Hz rTMS trains with an interval of 1 second at $90 \%$ of the resting motor threshold. The main targets included the left inferior frontal gyrus, left superior frontal gyrus, right inferior frontal gyrus, left dorsolateral prefrontal cortex, left and right anterior temporal lobe, and vertex. All patients with the non-fluent variant were tested in the left inferior frontal gyrus, left superior frontal gyrus, right inferior frontal gyrus, left dorsolateral prefrontal cortex, and vertex. All patients with the semantic variant were tested in the left anterior temporal lobe, right anterior temporal lobe, left dorsolateral prefrontal cortex, left inferior frontal gyrus, and vertex. Additional targets (i.e. supplementary motor area, anterior cingulate) were tested only in some patients. These regions were selected according to the previous knowledge about the most relevant regions in language and PPA (i.e. inferior frontal gyrus, anterior temporal lobe), previous targets in other brain stimulation studies in PPA (i.e. left dorsolateral prefrontal cortex), and regions with greater hypometabolism and atrophy in some patients (i.e. left superior frontal gyrus). Patients were evaluated immediately ( $<15$ minutes) after the rTMS session using the following language tasks: spontaneous speech, reading of 2 texts, object naming, and repetition (words and sentences). Patients with semantic variant PPA were also examined with a picture semantic association task. These tasks were developed by our group and include distinct but equivalent items according to several factors such as word frequency, length, degree of difficulty, etc. This procedure was used to avoid retest effects, and tests were different from those used as outcomes. Language testing was audio recorded and was evaluated by a blinded rater. Results were compared with baseline and between sessions to identify the most meaningful target for each patient. This target was decided by consensus between two of the investigators after evaluating the language changes scored by the blind rater. According to this strategy, patients received rTMS applied to the following areas: left inferior frontal gyrus (9 patients with nonfluent variant PPA), left superior frontal gyrus (3 patients with nonfluent variant PPA), left dorsolateral prefrontal cortex (1 patient with nonfluent variant PPA and 5 with semantic variant PPA), right superior frontal gyrus (1 patient with nonfluent variant PPA), and left anterior temporal lobe (1 patient with semantic variant PPA).

\section{4. rTMS protocol: target selection and control-site stimulation}


Brain stimulation was applied under neuronavigation, using a Magstim Rapid2 stimulator (Magstim, Whitland, UK) with a figure-eight coil. Patients received 15 sessions of $20-\mathrm{Hz}$ rTMS trains with an interval of 20 seconds at $100 \%$ of the resting motor threshold. A total of 1500 pulses were applied in each session. Control-site stimulation was applied over the vertex (27), with the following protocol: 600 pulses at $1 \mathrm{~Hz}$, with an interval of 10 seconds, at $25 \%$ of the resting motor threshold. Patients received 15 sessions on consecutive working days. All sessions were performed with online neuronavigation. In patients receiving both active- and control-site TMS, in the cross-over group, a washout period of at least 12 weeks was observed. These patients were informed that they would receive therapy using 2 different protocols.

\subsection{Outcomes and safety evaluation}

The primary outcome measure was the change in spontaneous speech (word count for 3 minutes using a story description task). Wordless children's books Frog Stories by Mercer Mayer were used (e.g. Frog goes to dinner) to elicit spontaneous speech. Participants were randomly assigned to tell one of the five books of the Frog Stories collection at baseline and after treatment. These stories are considered equivalents (28). The instruction given was: "This is a wordless book. Please tell us the story describing it in detail as if we could not see the book.". The test began when the examiner completed the instruction and finished at 3 minutes. No questions or interruptions by the examiner were allowed. The test was audiotaped and later transcribed for analysis.

Secondary outcomes were categorized as:

1) Language secondary outcomes:

i) Object naming test (96 items); the naming task was subdivided in 8 blocks. Each block comprised 12 pictures ranked by frequency of use (from most frequent to less). Items were line-drawn and included both animate and inanimate objects. Word frequency according to Zipf scale was $3.94 \pm 0.70$ (29). Mean age of acquisition was 4.27 \pm 1.32 (range 2.22-7.44), using an 11-point linear scale (value of 1 indicates an age less than 2 years-old, from 2 to 10 indicate learning ages of 2 to 10 years, and a value of 11 indicates that the word was learnt at 11 years or older (30). Imageability, familiarity, and concreteness were measured on a scale of 1 to 7 , with 7 being the most imageable, familiar, and concrete (31). Accordingly, mean imageability was $6.78 \pm 0.47$, familiarity was $5.99 \pm 0.69$, and concreteness was $6.03 \pm 0.47$. Mean number of phonemes was $6.04 \pm 1.71$, and mean number of syllables was $2.65 \pm 0.82$. Distributions of these parameters are shown in Supplementary Material Figure 1. Drawings of the nouns were presented using a computer, with the following instruction: "I will show several pictures. When you hear a sound, a picture will appear on the computer screen. Please name the picture using only one word". A maximum of 30 seconds was allowed for the subject's response and the test was audiotaped. Each item had only a correct response. If the patient gave an incorrect response, the examiner said, "No, tell me another name for that" or "That has another name". No phonological or semantic cues were given. The correct responses were not given in any case. A brief rest period was permitted between blocks, but not between items. 
ii) Story reading test (a text of 100 words. The text was presented in letter Cambria 22 in a single sheet of paper. Words were morphologically simple, with a mean of 1.94 syllables per word, and the frequency of nouns and adjectives was $4.23 \pm 0.84$ and frequency of verbs was $4.63 \pm 0.85$ according to Zipf scale (31). Readability according to the Flesch-Szigriszt score was 73.47 (an index of how difficult a document is to read on a scale from 100, extremely easy, to 0, very complex) and the Fernández-Huerta score was 77.69 (a Spanish adaptation of the former index). Parameters of readability were calculated using the INFLESZ software (32). Thus, the text is considered as "quite easy" according to these indexes. We calculated the accuracy (defined as the number of words correctly read), and efficiency (defined as the accuracy divided by the time taken to read, expressed as a percentage).

iii) Repetition of syllables (8 syllables, e.g. "pa”, “ta”), pairs of syllables (8 pairs, e.g. "pa-ma”, "na-ba”), non-words (8 non-words, e.g. "amiteso", "biboterana"), sentences (10 sentences of increasing length, from 3 words and 6 syllables to 10 words and 18 syllables), and multisyllabic words (for the assessment of dysdiadochokinesia). Each type of word was scored individually. These tasks belong to the Aphasia module of the "Test Barcelona revised", a standardized battery for neuropsychological and language assessment in Spanish (33).

2) Global cognition, evaluated with the Addenbrooke's Cognitive Examination III (22).

3) Perception of change: Changes in the impression of clinical change reported by the patient and principal caregiver (rated from $0=$ very much worse to $10=$ very much improved; $5=$ no change)

4) Behavioral changes, according to the Neuropsychiatric Inventory (NPI) (34).

5) Brain metabolism changes on FDG-PET images.

Outcomes were examined immediately (less than 2 weeks) before the start of TMS therapy and within the first 2 weeks after the completion of treatment. In the crossover group, outcomes were evaluated before and after the completion of each period (active-site or control-site stimulation).

To evaluate safety, patients and caregivers were asked about potential adverse effects, during each session of rTMS and at the end of the study. In addition, electroencephalography studies were performed prior to the onset of the rTMS sessions, after session 7, and at the end of the course of treatment for safety purposes. The electroencephalogram was assessed by an expert clinical neurophysiologist blind to the treatment.

\subsection{Neuroimaging preprocessing and analysis}

Statistical Parametric Mapping (SPM) software version 12 was used to preprocess and analyze the images. PET images were coregistered to T1-weighted MRI. Images were spatially normalized and subsequently smoothed at $8 \mathrm{~mm}$ full width at half maximum. Global intensity scaling was performed. A paired sample t-test was used to compare brain metabolism before and after active- and control-site rTMS. Age, sex, and site of stimulation were added as covariates. A threshold of family-wise error- 
corrected $p$-value $<0.05$ was used to correct for multiple comparisons. If non-statistically significant results were obtained, we used an uncorrected $p$-value $<0.001$ as an exploratory analysis. An extent threshold of $k=50$ was used.

A two-sample t-test was used to compare each diagnostic group (nonfluent and semantic PPA) versus a group of 40 healthy controls, using age and sex as covariates. A family-wise error corrected $p$-value $<0.05$ and $\mathrm{k}=50$ were used for multiple comparisons correction (Figure 3 ). This analysis was also conducted individually to represent the regions with brain hypometabolism for each patient and the targets used in each patient. In this case, an uncorrected $\mathrm{p}$-value $<0.001$ and an extent threshold of $\mathrm{k}=50$ voxels was used (Figure 4) (35).

\subsection{Sample size}

Given the lack of previous studies into the use of rTMS in patients with PPA, we estimated that a sample size of at least 15 participants was needed to obtain more than $80 \%$ power for detecting effect sizes larger than $d=0.80$ in the primary outcome.

\subsection{Standard protocol approvals, registrations, and patient consents}

The study was conducted with the approval of our hospital's Ethics Committee (study code: 17-247E) and in accordance with the Declaration of Helsinki and its subsequent amendments. The study was registered at clinicaltrials.gov (NCT03580954) (URL of the trial record registry: https://clinicaltrials.gov/ct2/show/NCT03580954). All patients (or their legally authorized representatives) gave written informed consent.

\subsection{Statistical analysis}

Statistical analysis was performed using version 20 of the IBM ${ }^{(R)}$ SPSS Statistics software. Descriptive results are shown as mean \pm standard deviation or frequency (percentage). Because the sample size was less than 30, we opted to use non-parametric tests. We used the Mann-Whitney $\mathrm{U}$ test for comparisons between 2 groups (active- and control-site rTMS), and the paired samples Wilcoxon test to evaluate changes within the same subjects before and after active-site TMS. Effect sizes were estimated with Hedges' $g$ and classified as small $(g=0.20)$, moderate $(g=0.50)$, or large $(g=0.80)$. A $p$-value $<0.05$ (two-tailed) was considered statistically significant.

Furthermore, a mixed three-way ANOVA was performed to understand the effects of rTMS on the outcomes of the study. The type of stimulation (active-site and control-site), and the clinical variant (nonfluent and semantic) were included as inter-subject factors in the analysis. Sidak method was used to correct for multiple comparisons. Accordingly, adjusted p-values are reported. Both numerical methods (skewness and kurtosis values and the Shapiro-Wilk test), and graphical methods (Normal Q-Q Plots) were used to measure normality. The outliers were kept in the analysis because they did not materially 
affect the results as assessed by a comparison of the results with and without the outliers. There was homogeneity of variances, as assessed by Levene's test for equality of variances $(p>0.05)$.

\section{Results}

\subsection{Effects of active-site rTMS compared with control-site rTMS}

We evaluated changes in language task performance between baseline and immediate post-treatment assessments by comparing patients receiving rTMS over the active site $(n=20)$ and those receiving rTMS over the vertex $(n=7)$. After rTMS, the active group scored higher for spontaneous speech (word count), reading accuracy, repetition of syllables, and pairs of syllables. The active group also presented higher scores in picture object naming.

Conversely, we did not find statistically significant changes in reading efficiency, repetition of non-words, repetition of sentences, or in the diadochokinetic task.

We also found a greater effect in the NPI total score, and in subscores for depression and apathy, as well as a trend toward statistical significance in anxiety. No statistically significant differences were observed between the 2 groups in the Addenbrooke's Cognitive Examination III (Table 2).

For all language tests, the NPI, and the Addenbrooke's Cognitive Examination III, no statistically significant differences were observed at baseline between the active- and control-site rTMS groups ( $p>0.10)$ (Supplementary Material Table S2).

\subsection{Effects of active-site rTMS compared with baseline}

We compared the results obtained in the different tests performed before and after active-site rTMS treatment in all 20 patients. Statistically significant differences were observed in the spontaneous speech task (word count), syllable repetition task. In addition, NPI scores were lower for the overall score and apathy, and a trend toward lower scores for depression. No statistically significant differences were observed in Addenbrooke's Cognitive Examination III scores. We did not find statistically significant changes in reading accuracy, reading efficiency, repetition of pairs of syllables, repetition of non-words, repetition of sentences, picture object naming, or the diadochokinetic task (SupplementaryTable S3).

\subsection{Perception of change}

A favorable clinical impression of change, as reported by patients and caregivers, was observed in the active group in comparison to controls $(p<0.001)$. In the active group, according to the patient's impression of change, $9(45 \%)$ reported a one-point increase on the scale, $4(20 \%)$ reported a 2-point increase, $2(10 \%)$ reported a 3-point increase, and $5(25 \%)$ showed no significant change. In the control group, 3 (42.90\%) reported no notable change; 1 (14.30\%) reported a one-point decrease, and 3 (42.90\%) reported a decrease of 2 points. Furthermore, caregivers reported an improvement of one point for 8 patients (40\%) in the active-site stimulation group, 2 points for 8 patients (40\%), 3 points for one $(5 \%)$, 
and 4 points for one (5\%), no significant change in $2(10 \%)$. In the control group, caregivers reported no changes in 3 cases $(42.9 \%)$ and a worsening of one point in the remaining cases (57.1\%). The kappa coefficient for agreement between patient and caregiver impression of change was 0.79 .

An impression of improvement was also detected when evaluating the change after the treatment in the active group in comparison to the control group (caregiver impression of change in active group $1.55 \pm 0.94$ vs $-0.57 \pm 0.53$ in the control group, $U=3.0, p<0.001$; patient impression of change in the active group $1.12 \pm 0.91$ vs. $-1.0 \pm 1.0$ in the control group, $U=7.5, p<0.001)$.

\subsection{Three-way ANOVA}

For the primary outcome, the three-way interaction between the change in spontaneous speech (word count), the type of stimulation (control site rTMS or active site rTMS), and the PPA variant were not statistically significant $(F(1,23)=1.25, p=0.275, \eta 2=0.052)$. There was a statistically significant twoway interaction between the primary outcome, and the type of stimulation $(F(1,23)=5.32, p=0.03)$. The other two-way interactions were not statistically significant $(p>0.05)$. After multiple comparisons correction, we found statistically significant differences in pre-post treatment primary outcome when active site rTMS was performed for the two clinical forms of PPA $(p<0.05)$ (Table 3$)$

Similarly, a three-way interaction was conducted for secondary clinical outcomes (Supplementary Material Table S4). Mauchly's test of sphericity indicated that the assumption of sphericity was violated. $\left(X^{2}(90)=1002,25, p<0.01\right)$. Therefore, Greenhouse-Geisser correction was applied. There was no statistically significant interaction between outcomes, rTMS sites and PPA clinical forms $(F(1.11,233.35)$ $=0.46, p=0.856$, partial $\eta^{2}=477.57$ ). After multiple comparisons correction, the group that received rTMS at the control site only had statistically significant differences in patient perception of change in the nonfluent PPA group with a worsening in the impression of change scale $(p<0.001)$. Regarding active rTMS, in the nonfluent variant statistically significant differences were observed in the repetition of pairs of syllables $(p=0.007)$ and in the patient and caregiver perception of change $(p<0.001)$, and a trend towards statistical significance in the repetition of syllables $(p=0.09)$ and the NPI depression scores $(p=0.05)$. Regarding semantic variant, differences with active TMS were obtained in the total NPI score $(p=0.010)$, in the caregiver's perception of change $(p=0.001)$ and a trend towards significance in the patient's perception of change both with higher post rTMS scores $(p=0.09)$.

\subsection{PET imaging findings}

In the group receiving active-site rTMS, the main regions showing higher metabolism after the treatment included left middle and superior temporal gyri, supramarginal gyrus, and superior and inferior parietal lobes; precuneus and posterior cingulate; left inferior frontal, medial frontal, and precentral gyri; bilateral superior and medial frontal gyri, and supplementary motor area; and left thalamus, insula, midbrain, and cerebellum (Figure 5; Supplementary Material Table S5). The inverse contrast (regions with increased metabolism in the PET scan acquired prior to treatment) did not report any significant cluster. SPM analysis of images from the control group (pre- vs post-control site stimulation) did not show any 
suprathreshold clusters. Using an uncorrected p-value $<0.001$ and extent threshold of $k=100$, we did not find any significant result.

\subsection{Treatment adherence and adverse effects}

All patients completed the treatment course. No severe adverse events were reported, and the treatment was well tolerated. Three patients (15\%), all in the active group, reported mild pain during the stimulation. One patient reported a mild headache after 3 sessions. No other adverse effects were reported. None of the electroencephalography studies performed during the study revealed epileptiform activity.

\section{Discussion}

In this study, we aimed to evaluate the effect of rTMS on PPA using several clinical and neuroimaging outcome measures covering different aspects of the disease. The most important finding is that rTMS over the active target improved performance in several language tasks. Specifically, we detected an increase in the number of words elicited during a spontaneous speech task, with a large effect size with respect to patients receiving rTMS over the control site. Improvements were also observed in comparison to baseline assessments, and independently from the clinical variant of PPA. Spontaneous speech and language production are valuable indicators in aphasias due to the participation of the main processes involved in language, including the generation of thoughts, word retrieval, semantics, grammar, phonology, and motor speech. In addition, language tasks regarded as secondary outcomes associated with phonology and motor speech, including repetition of syllables and pairs of syllables, also responded to the treatment when results were compared to those for control-site rTMS and baseline assessment. Similarly, confrontation object naming remained stable in the active group, whereas performance decreased in the control group. A positive effect was also observed in reading accuracy. Conversely, no significant effect was observed in dysdiadochokinesia, probably because this feature is observed only in a subgroup of patients.

Regarding the other secondary clinical endpoints, we found no statistically significant differences in global cognition, as measured by the Addenbrooke's Cognitive Examination III. Although this instrument has been validated for monitoring of patients with PPA, the rate of annual decline is estimated at 10 points per year, and the scale is probably not sufficiently sensitive over shorter periods (36). Interestingly, we observed an improvement in neuropsychiatric symptoms, and specifically in apathy and depression, with moderate and large effect sizes. This finding is noteworthy because apathy and depression are frequent in all PPA variants (37), and these behavioural disorders have consistently been associated with more significant functional impairment and poorer outcomes (38). A recent pilot study has also shown improvement of apathy in 9 patients with mild cognitive impairment treated with rTMS (39). Overall, these findings suggest that rTMS may be a potential treatment for apathy and/or depression in patients with PPA.

Another interesting and relevant result is the positive effect on the patient and caregiver global impression of change, which showed an effect of the treatment in almost all patients, and when 
compared against those receiving control-site stimulation. We used a 10-point scale rather than the traditional 7-point version, to facilitate understanding by patients and caregivers. These kinds of scale, although subjective, are considered reliable and adequate because they contribute real-life information (40), and may be more meaningful than other measures that capture only some specific aspects of the disease.

One of our most striking results is the detection of increased regional brain metabolism with rTMS. FDGPET is considered a reliable tool for evaluating synaptic activity. In this regard, synaptic failure is one of the hallmarks of neurodegenerative disorders, and the extent of hypometabolism correlates with cognitive function and disease stage. Previous studies have found metabolic changes during treatment with TMS, generally performed at the same time as the PET scan (41). However, to our knowledge, this is the first study evaluating FDG-PET findings after TMS therapy in a neurodegenerative disease. We found several clusters in both hemispheres, but predominantly in the left hemisphere. On the one hand, several of the regions identified correspond to the left parieto-temporal and frontal lobes, including the inferior frontal gyrus. These regions are mainly associated with the dorsal language network, suggesting phonological activation. Furthermore, other regions, including Broca's area and the precentral and bilateral supplementary motor areas, have been associated with motor speech and automation during speech. The activation of the dorsal stream found in our study suggests a different mechanism of improvement in non-fluent and semantic variants. Although both ventral and dorsal pathways are damaged in these variants to some degree (42), the dorsal stream is more damaged in the non-fluent variant, and TMS would activate this pathway directly. Conversely, in semantic PPA, the ventral stream is more severely impaired, and the activation of the dorsal pathway could compensate for the semantic dysfunction by activating the phonological loop. However, our findings are probably a consequence of the targets used in this study, which were more associated with the dorsal pathway, and further studies are necessary to elucidate potential differences in the mechanisms of improvement in brain stimulation across PPA variants. On the other hand, brain metabolism was also increased in several regions involving the bilateral precuneus and cingulate gyri, which may be explained by the activation of the default mode network. Besides their role in language, several of these regions have been associated with the pathophysiology of apathy in frontotemporal dementia syndromes (43). We also found higher metabolism in regions including the thalamus, putamen, midbrain, and cerebellum, which confirms that rTMS also recruited connections with subcortical regions. Our findings suggest that active-site rTMS is able to enhance synaptic activity in PPA, and modulates excitability not only in the target regions but also in other connected regions at both a cortical and a subcortical level. These findings may support the interest of applying rTMS protocols based on network-level connectivity, which could enhance the effect of TMS over conventional anatomically defined targets (44). In this regard, studies evaluating the capacity of rTMS to activate specific networks in patients with neurodegenerative disorders according to the baseline structural and functional connectivity are necessary.

All patients received high-frequency stimulation, which is consistent with previous studies suggesting that excitatory protocols might be more useful in neurodegenerative aphasia, whereas low-frequency protocols of the non-dominant hemisphere have been emphasized in post-stroke aphasia (45). Our study 
used a novel approach, with personalized targeting of stimulation based on the response of language parameters to a single session of rTMS. Because the extent of neurodegeneration is variable across patients with PPA, and cortical atrophy seems to follow connectional pathways arising from impaired regions (46-47), we hypothesize that certain brain regions may be more refractory to stimulation in some patients with more severe damage. As shown in Figure 4, regions impaired in each PPA patient are heterogeneous, and this fact may have importance in the TMS protocol design. Accordingly, stimulation of other nodes within the same network may be more feasible in some cases, while in other cases the stimulation of compensatory networks may be more appropriate to achieve positive effects. This is independent of the fact that there may be individual variability in language network localization between patients (48). In this regard, our study is a first attempt to develop a personalized protocol for rTMS in patients with PPA. Further studies with larger sample sizes are needed to define the baseline characteristics that are associated with a better response to each target. Future research should validate this approach by comparing personalized targeting with fixed protocols (i.e. inferior frontal gyrus or dorsolateral prefrontal cortex). In addition, the use of data-driven approaches may be useful in the selection of protocols according to the clinical, neuroimaging, and connectivity profile of each patient.

In this study, TMS therapy was performed independently of language training. Language training including the number of hours per week, sessions, protocol, etc. was not modified during the study. In addition, a relatively large percentage of patients did not receive language therapy (60\% in the active group and $43 \%$ in the control group). These findings may suggest a direct effect of TMS over language function. Previous research using single-session protocols in PPA supports this effect (49). Besides, studies in post-stroke aphasia have demonstrated an enhancement of phonological access using TMS (50). The increase of synaptic activity in PET observed in our study also supports a potential direct effect of rTMS, which may make language training more effective.

Our study has some limitations. First, the cross-over design was only performed in a subgroup of patients, and the study comprised a total of 20 patients with PPA. A larger sample size would be desirable in future studies. Second, clinical and neuroimaging assessments were performed shortly after the end of the therapy. The duration of the effect of rTMS in neurodegenerative disorders is not well known, and it is difficult to estimate due to the progressive nature of these disorders, but studies of transcranial direct current stimulation have found effects up to 2 months after the end of the therapy (17). Third, our study was designed to evaluate the effect on the whole group of PPA. For this reason, we used several language tasks that are invariably impaired in both the nonfluent and semantic variants of PPA, such as naming, spontaneous speech, or reading. However, a favorable response in the primary outcome was observed in both variants using ANOVA. More specific and sensitive tasks for each variant may be more appropriate in future studies specifically focused on one of these variants. In addition, the proportion of patients with each variant receiving control-site rTMS was not adequately balanced due to one patient with semantic variant PPA dropping out of the study. Fourth, we did not include patients with logopenic PPA, because clinical trials using disease-modifying therapies for AD (anti-amyloid, anti-tau drugs) were available. Thus, our findings are restricted to the non-fluent and semantic variant. However, previous 
studies using non-invasive brain stimulation have also detected positive effects in logopenic aphasia (17).

In conclusion, our study suggests that high-frequency rTMS with personalized targeting improves language, apathy, and depression, but not global cognition in patients with PPA. FDG-PET performed after rTMS also revealed an increase in regional brain metabolism, which suggests an enhancement of synaptic activity with the treatment. This study provides a rationale and supports the consideration for conducting a phase III randomized clinical trial using rTMS in PPA.

\section{Abbreviations}

$-A D$ : Alzheimer's disease

-FDG-PET: 18F-Fluorodeoxyglucose positron emission tomography

-MRI: magnetic resonance imaging.

-NIBS: non-invasive brain stimulation

-NPI: Neuropsychiatric Inventory

-PPA: Primary Progressive Aphasia.

-rTMS: repetitive Transcranial Magnetic Stimulation.

-SPM: Statistical Parametric Mapping

-tDCS: transcranial direct current stimulation

\section{Declarations}

\section{Ethical approval and consent to participate}

The study was conducted with the approval of our hospital's Ethics Committee (study code: 17-247E) and in accordance with the Declaration of Helsinki and its subsequent amendments. The study was registered at clinicaltrials.gov (NCT03580954). All patients (or their legally authorized representatives) gave written informed consent.

Consent for publication: Not applicable.

Availability of data and materials: The datasets used and/or analysed during the current study are available from the corresponding author on reasonable request.

Competing interests: The authors declare that they have no competing interests 
Funding: The research group was provided with a research support technician by the Instituto de Salud Carlos III (PTA 17/13618).

\section{Author's contributions:}

-Conceptualization and design of the study: VP, JLA, JMG, JAMG.

-Data curation: VP, MNCM, ADA, CDA, MY, MTC.

-Formal analysis: VP, ADA, JAMG, JLA.

-Funding acquisition: JMG, JAMG, TMR.

-Investigation: VP, MNCM, ADA, CDA, MY, MTC, TMR, JLC, JAMG.

-Methodology: VP, JMG, JLA, JAMG.

-Supervision: JMG, JLC.

-Writing original draft: VP, JAMG.

-Writing review and editing:: JMG, MTC, JLA.

\section{Acknowledgements}

We are grateful to all the participants and their caregivers for their interest in our study. We would like to thank Dr Joaquín Ojeda from Hospital Infanta Sofía, and Dr. María José Gil Moreno, for his collaboration. We would also like to thank Helena Briales of "Asociación Ayuda Afasia" for her help and her commitment to the patients.

\section{Conflicts of Interest}

Nothing to report.

\section{References}

1. Gorno-Tempini ML, Hillis AE, Weintraub S, Kertesz A, Mendez M, Cappa SF, et al. Classification of primary progressive aphasia and its variants. Neurology. 2011;76(11):1006-14.

2. Tee BL, Gorno-Tempini ML. Primary progressive aphasia: a model for neurodegenerative disease. Curr Opin Neurol. 2019;32(2):255.

3. Bergeron D, Gorno-Tempini ML, Rabinovici GD, Santos-Santos MA, Seeley W, Miller BL, et al. Prevalence of amyloid- $\beta$ pathology in distinct variants of primary progressive aphasia. Ann Neurol. 2018;84(5):729-40. 
4. Botha H, Duffy JR, Whitwell JL, Strand EA, Machulda MM, Schwarz CG, et al. Classification and clinicoradiologic features of primary progressive aphasia (PPA) and apraxia of speech. Cortex. $2015 ; 69,: 220-36$.

5. Matias-Guiu JA, Díaz-Álvarez J, Cuetos F, Cabrera-Martín MN, Segovia-Ríos I, Pytel V, et al. Machine learning in the clinical and language characterisation of primary progressive aphasia variants. Cortex. 2019;119:312-23.

6. Burke MJ, Fried PJ, Pascual-Leone A. Transcranial magnetic stimulation: Neurophysiological and clinical applications. Handb Clin Neurol. 2019;163:73-92.

7. Málly J. Non-invasive brain stimulation (rTMS and tDCS) in patients with aphasia: mode of action at the cellular level. Brain research bulletin. 2013;98:30-5.

8. Lefaucheur JP, Aleman A, Baeken C, Benninger DH, Brunelin J, Di Lazzaro V, et al. Evidence-based guidelines on the therapeutic use of repetitive transcranial magnetic stimulation (rTMS): an update (2014-2018). Clinical neurophysiology. 2020;131(2):474-528.

9. Dong X, Yan L, Huang L, Guan X, Dong C, Tao H, et al. Repetitive transcranial magnetic stimulation for the treatment of Alzheimer's disease: A systematic review and meta-analysis of randomized controlled trials. PloS one. 2018;13(10):e0205704.

10. Benussi A, Grassi M, Palluzzi F, Koch G, Di Lazzaro V, Nardone R, et al. Classification accuracy of transcranial magnetic stimulation for the diagnosis of neurodegenerative dementias. Ann Neurol. 2020;87(3):394-404.

11. Antczak J, Kowalska K, Klimkowicz-Mrowiec A, Wach B, Kasprzyk K, Banach M, ... Słowik A. Repetitive transcranial magnetic stimulation for the treatment of cognitive impairment in frontotemporal dementia: an open-label pilot study. Neuropsychiatr Dis Treat. 2018;14:749.

12. Margolis SA, Festa EK, Papandonatos GD, Korthauer LE, Gonsalves MA, Oberman L, et al. A pilot study of repetitive transcranial magnetic stimulation in primary progressive aphasia. Brain Stimul. 2019;12(5):1340-2.

13. Cotelli M, Manenti R, Petesi M, Brambilla M, Cosseddu M, Zanetti O, et al. Treatment of primary progressive aphasias by transcranial direct current stimulation combined with language training. $J$ Alzheimers Dis. 2014;39(4):799-808.

14. de Aguiar V, Zhao Y, Faria A, Ficek B, Webster KT, Wendt H, et al. Brain volumes as predictors of tDCS effects in primary progressive aphasia. Brain Lang. 2020;200:104707.

15. Gervits F, Ash S, Coslett HB, Rascovsky K, Grossman M, Hamilton R. Transcranial direct current stimulation for the treatment of primary progressive aphasia: An open-label pilot study. Brain Lang. 2016;162:35-41.

16. Teichmann M, Lesoil C, Godard J, Vernet M, Bertrand A, Levy R, et al. Direct current stimulation over the anterior temporal areas boosts semantic processing in primary progressive aphasia. Ann Neurol. 2016;80(5):693-707.

17. Tsapkini K, Webster KT, Ficek BN, Desmond JE, Onyike CU, Rapp B, et al. Electrical brain stimulation in different variants of primary progressive aphasia: A randomized clinical trial. Alzheimer's dementia 
NY. 2018;4:461-72.

18. Cotelli M, Manenti R, Paternico D, Cosseddu M, Brambilla M, Petesi M, et al. Grey matter density predicts the improvement of naming abilities after tDCS intervention in agrammatic variant of primary progressive aphasia. Brain Topogr. 2016;29(5):738-51.

19. Routier A, Habert MO, Bertrand A, Kas A, Sundqvist M, Mertz J, et al. Structural, microstructural, and metabolic alterations in primary progressive aphasia. Frontiers in Neurology. 2018;9:766.

20. Landin-Romero R, Tan R, Hodges JR, Kumfor F. An update on semantic dementia: genetics, imaging, and pathology. Alzheimer's Research Therapy. 2016;8:52.

21. Tetzloff KA, Duffy JR, Clark HM, Strand EA, Machulda MM, Schwarz CG, et al. Longitudinal structural and molecular neuroimaging in agrammatic primary progressive aphasia. Brain. 2018;141:302-17.

22. Matias-Guiu JA, Cortés-Martínez A, Valles-Salgado M, Rognoni T, Fernández-Matarrubia M, MorenoRamos T, et al. Addenbrooke's cognitive examination III: diagnostic utility for mild cognitive impairment and dementia and correlation with standardized neuropsychological tests. International psychogeriatrics. 2017;29(1):105-13.

23. Sapolsky D, Bakkour A, Negreira A, Nalipinski P, Weintraub S, Mesulam MM, et al. Cortical neuroanatomic correlates of symptom severity in primary progressive aphasia. Neurology. 2010;75(4):358-66.

24. Knopman DS, Weintraub S, Pankratz VS. Language and behavior domains enhance the value of the clinical dementia rating scale. Alzheimer's Dement. 2011;7(3):293-9.

25. Matías-Guiu JA, Cuetos F, Cabrera-Martín MN, Valles-Salgado M, Moreno-Ramos T, Carreras JL, et al. Reading difficulties in primary progressive aphasia in a regular language-speaking cohort of patients. Neuropsychologia. 2017;101:132-40.

26. Varrone A, Asenbaum S, Vander Borght T, Booij J, Nobili F, Någren K, et al. EANM procedure guidelines for PET brain imaging using [18 F] FDG, version 2. Eur J Nucl Med Mol Imaging. 2009;36(12):2103.

27. Jung J, Bungert A, Bowtell R, Jackson SR. Vertex Stimulation as a Control Site for Transcranial Magnetic Stimulation: A Concurrent TMS/fMRI Study. Brain Stimul. 2016;9(1):58-64.

28. Heilmann JJ, Rojas R, Iglesias A, Miller JF. Clinical impact of wordless picture storybooks on bilingual narrative language production: a comparison of the "Frog" stories. International Journal of Language Communication Disorders. 2016;51(3):339-45.

29. Van Heuven WJB, Mandera P, Keuleers E, Brysbaert M, Subtlex. -UK: a new and improved word frequency database for British English. Quarterly Journal of Experimental Psychology. 2014;67(6):1176-90.

30. Alonso MA, Fernández A, Díez E. Subjective age-of-acquisition norms for 7,039 Spanish words. Behav Res Methods. 2015;47:268-74.

31. Duchon A, Perea M, Sebastián-Gallés N, Martí A, Carreiras M. EsPal: one-stop shopping for Spanish word properties. Behav Res Methods. 2015;45:1246-58. 
32. Barrio I, El programa I. 2015. Available from: https://legibilidad.blogspot.com/2015/01/el-programainflesz.html.

33. Peña-Casanova J. Programa integrado de exploración neuropsicológica: Test Barcelona Revisado. Barcelona: Masson; 2005.

34. Cummings JL, Mega M, Gray K, Rosenberg-Thompson S, Carusi DA, Gornbein. J The Neuropsychiatric Inventory: comprehensive assessment of psychopathology in dementia Neurology. 1994;44(12):2308-14.

35. Matias-Guiu JA, Cabrera-Martín MN, Pérez-Castejón MJ, Moreno-Ramos T, Rodríguez-Rey C, GarcíaRamos R, et al. Visual and statistical analysis of 18F-FDG PET in primary progressive aphasia. Eur $\mathrm{J}$ Nucl Med Mol Imaging. 2015;42(6):916-27.

36. Leyton CE, Hornberger M, Mioshi E, Hodges JR. Application of Addenbrooke's cognitive examination to diagnosis and monitoring of progressive primary aphasia. Dement Geriatr Cogn Disord. 2010;29(6):504-9.

37. Singh TD, Duffy JR, Strand EA, Machulda MM, Whitwell JL, Josephs KA. Neuropsychiatric symptoms in primary progressive aphasia and apraxia of speech. Dement Geriatr Cogn Disord. 2015;39:22838.

38. Lansdall CJ, Coyle-Gilchrist IT, Rodríguez PV, Wilcox A, Wehmann E, Robbins TW, et al. Prognostic importance of apathy in syndromes associated with frontotemporal lobar degeneration. Neurology. 2019;92(14):e1547-57.

39. Padala PR, Padala KP, Lensing SY, Jackson AN, Hunter CR, Parkes CM, et al. Repetitive transcranial magnetic stimulation for apathy in mild cognitive impairment: a double-blind, randomized, shamcontrolled, cross-over pilot study. Psychiatry research. 2018;261:312-8.

40. Robert P, Ferris S, Gauthier S, Ihl R, Winblad B, Tennigkeit F. Review of Alzheimer's disease scales: is there a need for a new multi-domain scale for therapy evaluation in medical practice? Alzheimer's research therapy. 2010;2(4):24.

41. Siebner HR, Peller M, Willoch F, Minoshima S, Boecker H, Auer C, et al. Lasting cortical activation after repetitive TMS of the motor cortex: a glucose metabolic study. Neurology. 2000;54(4):956-63.

42. Agosta F, Galantucci S, Canu E, Cappa SF, Magnani G, Franceschi M,et al. Disruption of structural connectivity along the dorsal and ventral language pathways in patients with nonfluent and semantic variant primary progressive aphasia: a DT MRI study and a literature review. Brain Lang. 2013;127(2):157-66.

43. Fernández-Matarrubia M, Matías-Guiu JA, Cabrera-Martín MN, Moreno-Ramos T, Valles-Salgado M, Carreras $\mathrm{JL}$, et al. Different apathy clinical profile and neural correlates in behavioral variant frontotemporal dementia and Alzheimer's disease. Int J Geriatr Psychiatry. 2018;33(1):141-50.

44. Momi D, Ozdemir RA, Tadayon E, Boucher P, Shafi MM, Pascual-eone A, et al. Network-level macroscale structural connectivity predicts propagation of transcranial magnetic stimulation. Neurolmage. 2021;229:117698. 
45. Norise $\mathrm{C}$, Hamilton RH. Non-invasive brain stimulation in the treatment of post-stroke and neurodegenerative aphasia: parallels, differences, and lessons learned. Frontiers in Human Neuroscience. 2017;10:675.

46. Collins JA, Montal V, Hochberg D, Quimby M, Mandelli ML, Makris N, et al. Focal temporal pole atrophy and network degeneration in semantic variant primary progressive aphasia. Brain. 2017;140(2):457-71.

47. Montembeault M, Chapleau M, Jarret J, Boukadi M, Laforce Jr R, Wilson MA, et al. Differential language network functional connectivity alterations in Alzheimer's disease and the semantic variant of primary progressive aphasia. Cortex. 2019;117:284-98.

48. Forkel SJ, Thiebaut de Schotten M, Dell'Acqua F, Kalra L, Murphy DG, Williams SC, et al. Anatomical predictors of aphasia recovery: a tractography study of bilateral perisylvian language networks. Brain. 2014;137(7):2027-39.

49. Cotelli M, Manenti R, Alberici A, Brambilla M, Cosseddu M, Zanetti O, et al. Prefrontal cortex rTMS enhances action naming in progressive non-fluent aphasia. Eur J Neurol. 2012;19:1404-12.

50. Harvey DY, Mass JA, Shah-Basak PP, Wurzman R, Faseyitan O, Sacchetti DL, et al. Continuous theta burst stimulation over right pars triangularis facilitates naming abilities in chronic post-stroke aphasia by enhancing phonological access. Brain Lang. 2019;192:25-34.

\section{Tables}

Table 1. Main clinical and demographic characteristics of the participants. Comparison between activeand control-site rTMS groups. 


\begin{tabular}{|c|c|c|c|}
\hline & $\begin{array}{l}\text { Active-site rTMS } \\
(n=20)\end{array}$ & $\begin{array}{l}\text { Control-site rTMS } \\
(n=7)\end{array}$ & $\begin{array}{l}\chi^{2} \text { or U } \\
\text { (p-value) }\end{array}$ \\
\hline Age (years) & $66.95 \pm 7.24$ & $66.14 \pm 7.31$ & $U=64.00(0.76)$ \\
\hline $\begin{array}{l}\text { Sex Male } \\
\text { Female }\end{array}$ & $\begin{array}{l}8(40 \%) \\
12(60 \%)\end{array}$ & $\begin{array}{l}3(42.90 \%) \\
4(67.1 \%)\end{array}$ & $\chi^{2}=0.02(1.00)$ \\
\hline Education (years) & $13.40 \pm 4.38$ & $13.86 \pm 3.18$ & $U=68.50(0.93)$ \\
\hline $\begin{array}{l}\text { PPA clinical type nfvPPA } \\
\text { svPPA }\end{array}$ & $\begin{array}{l}14(70 \%) \\
6(30 \%)\end{array}$ & $\begin{array}{l}6(85.70 \%) \\
1(14.30 \%)\end{array}$ & $\chi^{2}=0.66(0.63)$ \\
\hline Symptom duration (years) & $3.80 \pm 3.90$ & $3.71 \pm 1.79$ & $U=59.50(0.55)$ \\
\hline Language therapy & $8(40 \%)$ & $4(57.10 \%)$ & $\chi^{2}=0.61(0.66)$ \\
\hline ACE-III total score (maximum 100) & $64.80 \pm 22.93$ & $69.00 \pm 19.81$ & $U=63.00(0.69)$ \\
\hline ACE-III attention (maximum 18) & $13.40 \pm 4.96$ & $13.57 \pm 3.77$ & $U=64.00(0.73)$ \\
\hline ACE-III memory (maximum 26) & $16.65 \pm 8.06$ & $18.28 \pm 7.40$ & $U=59.00(0.54)$ \\
\hline ACE-III language (maximum 26) & $17.05 \pm 7.43$ & $19.57 \pm 4.31$ & $U=61.50(0.63)$ \\
\hline ACE-III fluency (maximum 14) & $5.20 \pm 3.65$ & $4.14 \pm 3.62$ & $U=60.50(0.59)$ \\
\hline PASS Rating & $7.45 \pm 4.31$ & $6.78 \pm 2.97$ & $\begin{array}{l}U=67.00 \\
(0.8)\end{array}$ \\
\hline FAQ Score & $4.25 \pm 4.33$ & $4.00 \pm 5.47$ & $\begin{array}{l}U=63 \\
(0.72)\end{array}$ \\
\hline FTD-CDR & $3.00 \pm 2.46$ & $2.71 \pm 2.03$ & $\begin{array}{l}U=69 \\
(0.95)\end{array}$ \\
\hline NPI Total score & $13.45 \pm 16.42$ & $9.42 \pm 9.34$ & $U=69.00(0.95)$ \\
\hline \multicolumn{4}{|c|}{$\begin{array}{l}\text { ACE-III: Addenbrooke's Cognitive Examination III; FAQ: Functional Activities Questionnaire; FTD-CDR: } \\
\text { Frontotemporal Dementia Modified Clinical Dementia Rating Scale; nfvPPA: nonfluent variant PPA; } \\
\text { NPI: Neuropsychiatric Inventory; PASS: Progressive Aphasia Severity Score; PPA: primary progressive } \\
\text { aphasia; SvPPA: semantic variant PPA; TMS: transcranial magnetic stimulation; U: Mann-Whitney U } \\
\text { test; } X^{2} \text { : chi-squared test. }\end{array}$} \\
\hline
\end{tabular}

Table 2. Effects of active-site rTMS compared with control-site rTMS. 


\begin{tabular}{|c|c|c|c|c|}
\hline \multirow[t]{2}{*}{ Outcomes } & \multicolumn{2}{|c|}{$\begin{array}{l}\text { Difference between post-intervention and } \\
\text { baseline }\end{array}$} & \multirow[t]{2}{*}{$\begin{array}{l}\mathrm{p}- \\
\text { value }\end{array}$} & \multirow[t]{2}{*}{$\begin{array}{l}\text { Hedges'g [Cl } \\
95 \%]\end{array}$} \\
\hline & $\begin{array}{l}\text { Active-site rTMS } \\
(n=20)\end{array}$ & $\begin{array}{l}\text { Control-site rTMS } \\
(n=7)\end{array}$ & & \\
\hline Word count * & $27.89 \pm 34.59$ & $-3.42 \pm 21.85$ & 0.02 & $\begin{array}{l}0.9 \\
{[0.06,1.88]}\end{array}$ \\
\hline Reading accuracy * & $20.55 \pm 64.60$ & $-28.14 \pm 29.05$ & 0.008 & $\begin{array}{l}0.81 \\
{[-0.60,1.72]}\end{array}$ \\
\hline Reading efficiency & $18.33 \pm 57.74$ & $-1.68 \pm 15.97$ & 0.49 & - \\
\hline Repetition of syllables * & $0.58 \pm 1.07$ & $-0.57 \pm 1.13$ & 0.03 & $\begin{array}{l}1.03 \\
{[0.13,1.96]}\end{array}$ \\
\hline $\begin{array}{l}\text { Repetition of pairs of } \\
\text { syllables * }\end{array}$ & $0.66 \pm 1.84$ & $-0.50 \pm 0.54$ & 0.03 & $\begin{array}{l}0.68 \\
[0.24,1.64])\end{array}$ \\
\hline Repetition of non-words & $-0.38 \pm 1.53$ & $-1.16 \pm 0.98$ & 0.13 & - \\
\hline Repetition of sentences & $-1.47 \pm 6.76$ & $-4.85 \pm 12.50$ & 0.96 & - \\
\hline Diadochokinetic task & $0.84 \pm 3.56$ & $-0.85 \pm 2.73$ & 0.11 & - \\
\hline Picture object naming * & $-0.23 \pm 7.50$ & $-4.28 \pm 8.01$ & 0.04 & $\begin{array}{l}0.52 \\
{[-0.34,1.40]}\end{array}$ \\
\hline ACE-III & $-2.20 \pm 7.32$ & $-0.57 \pm 2.87$ & 0.73 & - \\
\hline NPI total scores * & $-6.50 \pm 8.23$ & $2.00 \pm 3.16$ & 0.004 & $\begin{array}{l}1.12 \\
{[2.06,-0.22]}\end{array}$ \\
\hline Apathy * & $-1.90 \pm 2.78$ & $0.00 \pm 3.78$ & 0.03 & $\begin{array}{l}0.60 \\
{[-1.49,0.26]}\end{array}$ \\
\hline Depression * & $-1.25 \pm 3.07$ & $1.14 \pm 1.95$ & 0.03 & $\begin{array}{l}0.82 \\
{[-1.72,0.05]}\end{array}$ \\
\hline Anxiety & $-0.80 \pm 2.70$ & $0.57 \pm 0.97$ & 0.05 & $\begin{array}{l}0.55 \\
{[-1.44,0.30]}\end{array}$ \\
\hline $\begin{array}{l}\text { ACE-III: Addenbrooke's Co } \\
\text { Inventory. * Statistically s }\end{array}$ & $\begin{array}{l}\text { itive Examination } \\
\text { lificant mean dift }\end{array}$ & $\begin{array}{l}\text { : Confidence Interv } \\
\text { e. }\end{array}$ & Neurc & ychiatric \\
\hline
\end{tabular}

Table 3. Mixed three-way ANOVA for the primary outcome. Type of stimulation (active vs control-site), and PPA variant were included as factors in the statistical model. 


\begin{tabular}{|c|c|c|c|c|c|c|c|c|}
\hline \multirow[t]{2}{*}{ rTMS site } & \multirow[t]{2}{*}{$\begin{array}{l}\text { PPA clinical } \\
\text { form }\end{array}$} & \multirow{2}{*}{\multicolumn{2}{|c|}{ WC }} & \multirow[t]{2}{*}{$\begin{array}{l}\text { Differences in } \\
\text { averages }\end{array}$} & \multirow[t]{2}{*}{$\begin{array}{l}\text { Standard } \\
\text { error }\end{array}$} & \multirow[t]{2}{*}{$\begin{array}{l}\mathrm{p}- \\
\text { value }\end{array}$} & \multicolumn{2}{|c|}{$\begin{array}{l}\text { Confidence } \\
\text { interval }\end{array}$} \\
\hline & & & & & & & $\begin{array}{l}\text { Lower } \\
\text { limit }\end{array}$ & $\begin{array}{l}\text { Upper } \\
\text { limit }\end{array}$ \\
\hline \multirow{2}{*}{$\begin{array}{l}\text { Control site- } \\
\text { rTMS }\end{array}$} & nfv-PPA & 1 & 2 & 4.00 & 11,265 & 0.726 & $-19,30$ & 27,30 \\
\hline & sv-PPA & 1 & 2 & $2.842 \mathrm{E}-014$ & 27,594 & 1,000 & $-57,08$ & 57,08 \\
\hline \multirow{2}{*}{$\begin{array}{l}\text { Active site } \\
\text { rTMS }\end{array}$} & nfv-PPA & 1 & 2 & $-15.43^{*}$ & 7,375 & 0.048 & $-30,68$ &,- 17 \\
\hline & sv-PPA & 1 & 2 & $-56.00^{\star}$ & 11,265 & 0.000 & $-79,30$ & $-32,69$ \\
\hline \multicolumn{9}{|c|}{ Sidak test was used for multiple comparisons correction. } \\
\hline $\begin{array}{l}\text { PPA: prima } \\
\text { Pre rTMS; } 2\end{array}$ & $\begin{array}{l}\text { gressive ap } \\
\text { rTMS. *St }\end{array}$ & & & $\begin{array}{l}\text { no fluent } P \\
\text { ficant mean }\end{array}$ & $\begin{array}{l}\text { A: sen } \\
\text { ee. }\end{array}$ & & & Int. 1: \\
\hline
\end{tabular}

\section{Figures}

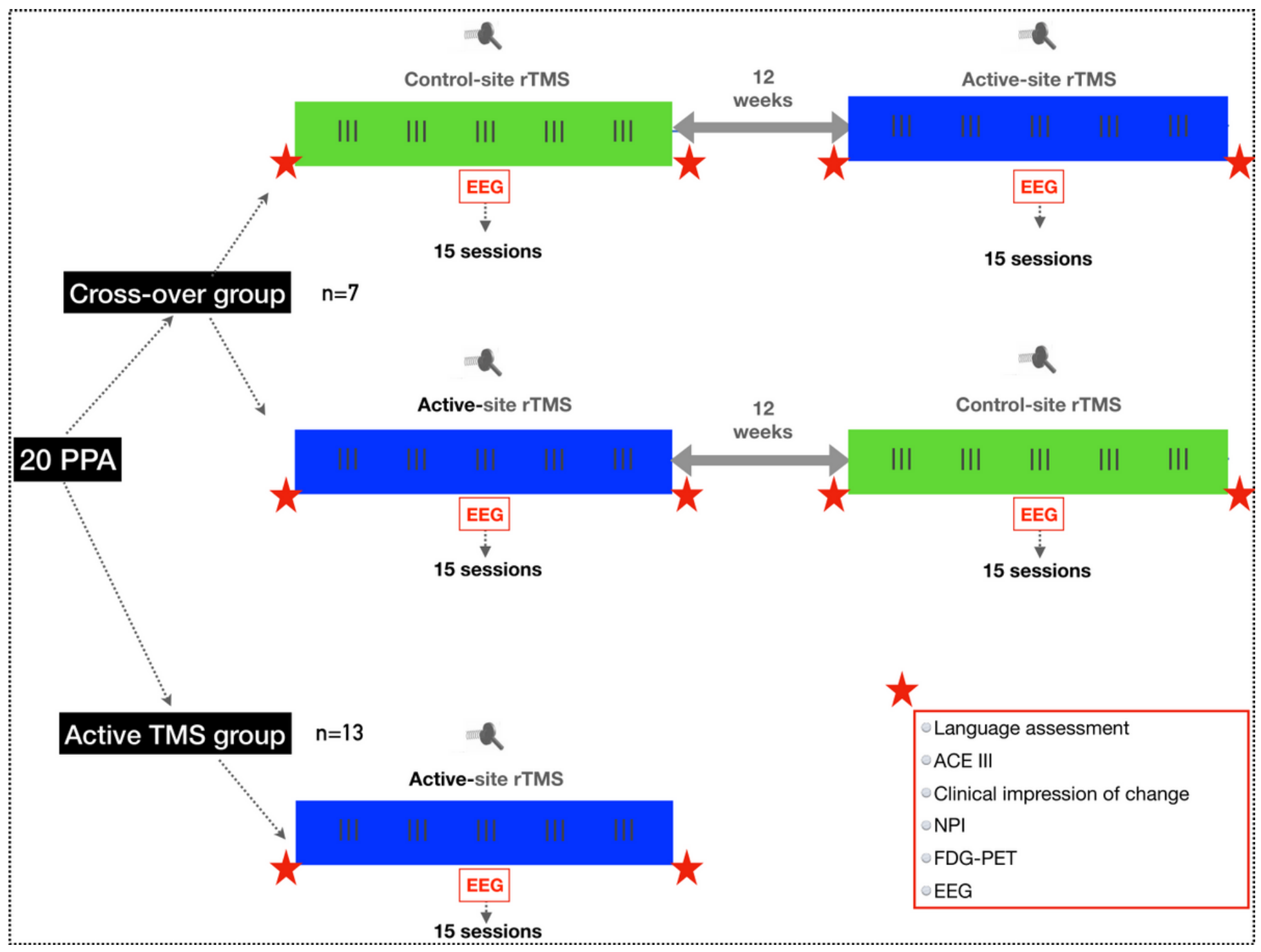


Figure 1

Study design. Patients were randomized at a 3:2 ratio into 2 groups: i) active-site rTMS only $(n=13)$; ii) a cross-over group $(n=7)$, in which patients were randomly assigned 1:1 to receive active-site rTMS followed by control-site rTMS, or vice versa.

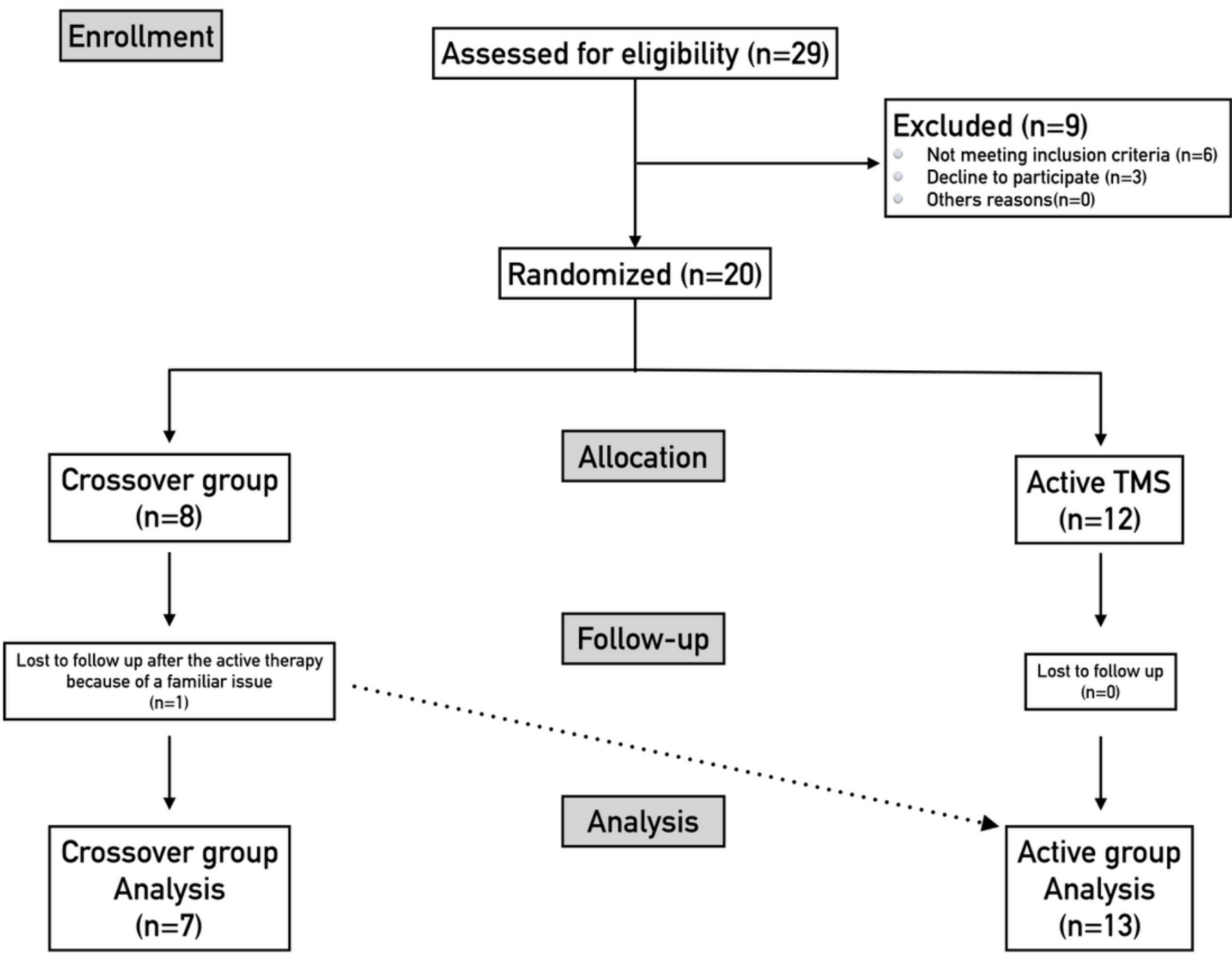

Figure 2

Flow diagram. 


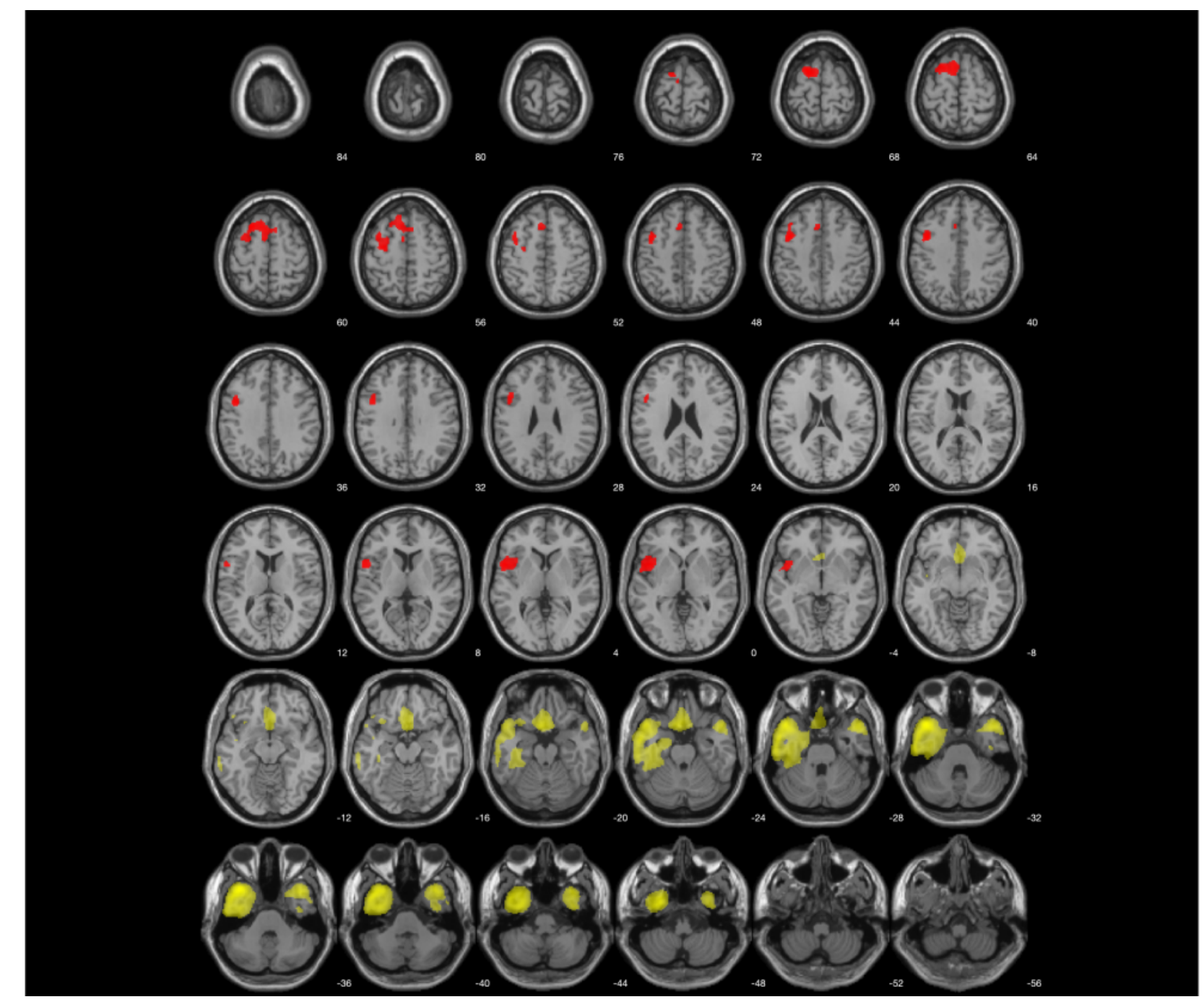

\section{Figure 3}

Regions with lower metabolism in the non-fluent and semantic PPA variants. Images are displayed on an MRI template. Nonfluent PPA (red) and semantic PPA (yellow) were compared with healthy controls using a two-sample t-test with a family-wise error corrected $p$-value $<0.05$. Images are shown using neurological orientation. 


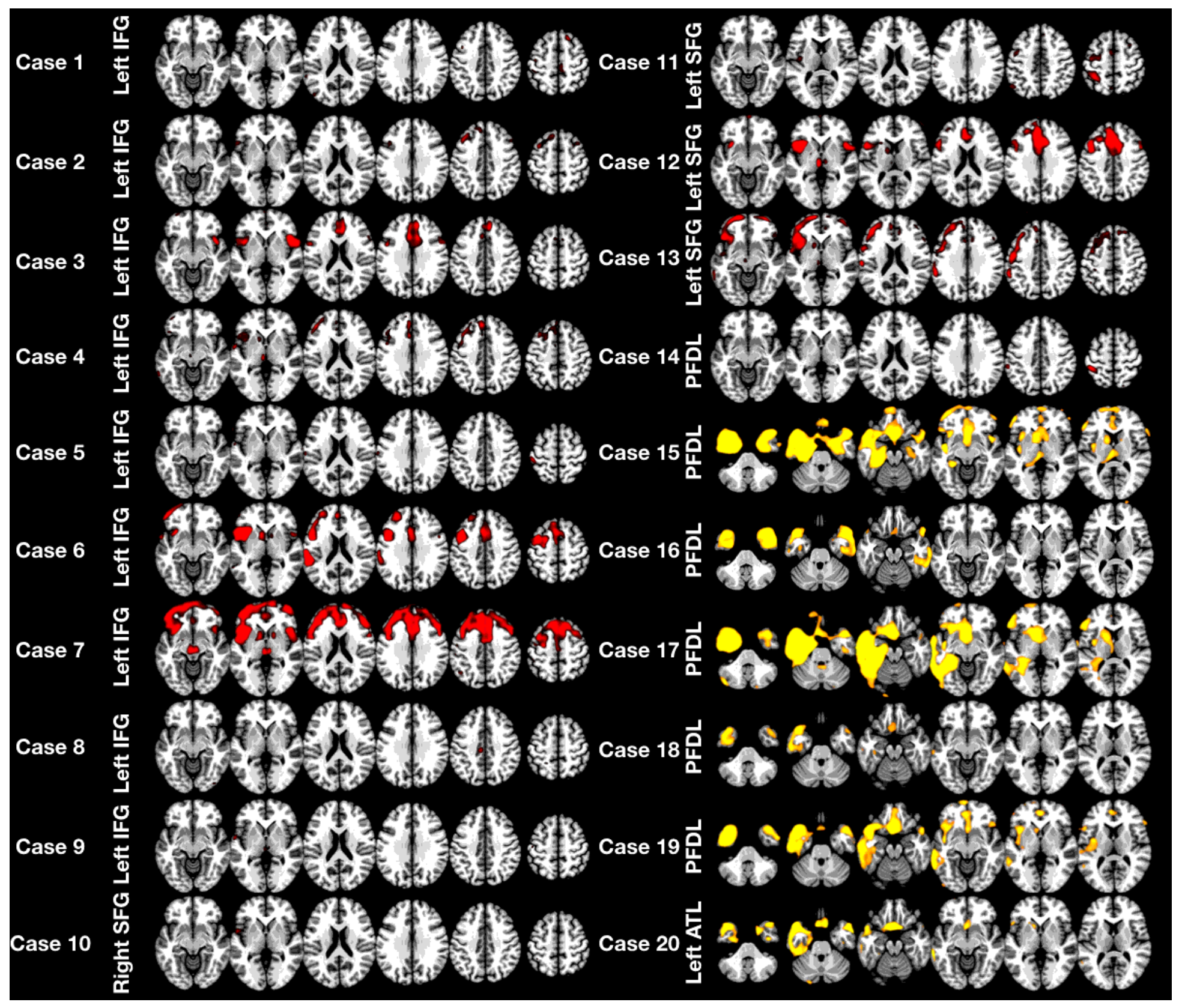

Figure 4

Regions with lower metabolism in each patient in comparison to the healthy control group. Targets used in each case are shown. Nonfluent PPA patients are represented in red, and semantic PPA in yellow. A two-sample t-test with an uncorrected p-value $<0.001$ was used (T-score threshold was set at 3.33, and maximum T-score was 5.0). Images are shown using neurological orientation. IFG: inferior frontal gyrus; SFG: superior frontal gyrus; PFDL: Left prefrontal dorsolateral cortex; ATL: anterior temporal lobe. 
$-24$

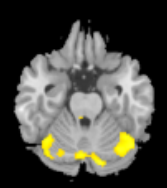

14

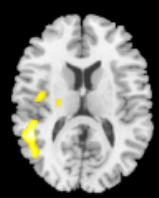

34

38

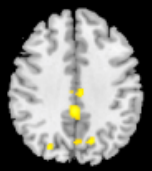

$-4$

18
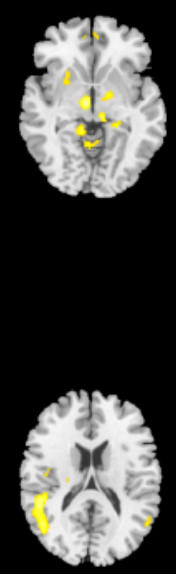

22
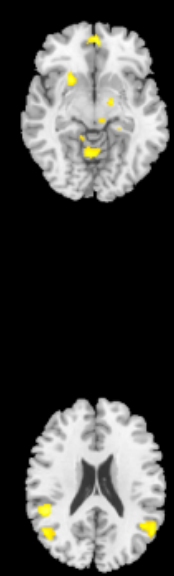

42
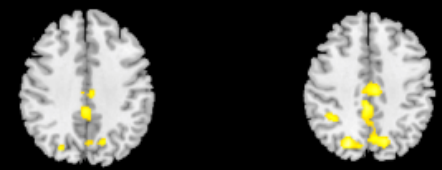

4

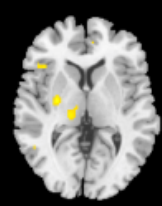

26

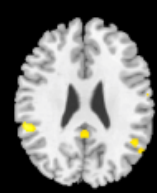

46
30

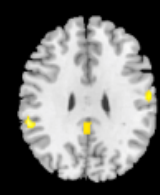

10

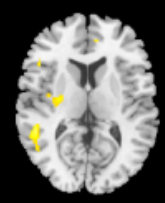

52

\section{Figure 5}

FDG-PET imaging results. Regions with higher metabolism after active-site rTMS in comparison to baseline (family-wise error-corrected p-value < 0.05). Age, sex, and site of stimulation were added as covariates. Images are shown using neurological orientation.

\section{Supplementary Files}

This is a list of supplementary files associated with this preprint. Click to download.

- S1Fig1AZipf.pdf

- S1Fig1BAoA.pdf

- S1Fig1CImageability.pdf

- S1Fig1DFamiliarity.pdf

- S1Fig1EConcreteness.pdf

- S1Fig1FNumberOfPhonemes.pdf 
- S1Fig1GNumberOfSyllables.pdf

- Tables1.docx

- Tables2.docx

- Tables3.docx

- TableS4.docx

- TableS5.docx 